Volume 31 (2022) 38-48

DOI: $10.24330 /$ ieja. 1058393

\title{
COREGULAR SEQUENCES AND TOP LOCAL HOMOLOGY MODULES
}

\author{
Nguyen Minh Tri \\ Received: 7 August 2020; Accepted: 8 October 2021 \\ Communicated by A. Çiğdem Özcan
}

\begin{abstract}
In this paper, we show that if $M$ is a non-zero Artinian $R$-module and $\underline{x}:=x_{1}, \ldots, x_{n}$ is an $M$-coregular sequence, then $x_{1}, \ldots, x_{n}$ is a $D\left(H \frac{x}{n}(M)\right)$ coregular sequence. Moreover, if $R$ is complete with respect to $I$-adic topology and $d=\operatorname{Ndim} M$, then $\operatorname{dim} H_{d}^{I}(M) \leq d$ and $\operatorname{depth} H_{I}^{d}(M) \geq \min \{2, d\}$ whenever $H_{d}^{I}(M) \neq 0$.
\end{abstract}

Mathematics Subject Classification (2020): 13C14, 13C15, 13D07

Keywords: Coregular sequence, local homology, Co-Cohen-Macaulay module

\section{Introduction}

In this paper, $R$ is a Noetherian commutative ring with identity, $I$ is an ideal of $R$ and $M$ is an $R$-module. The theory of local homology was initiated by Matlis [10] in 1974. This theory was studied and improved by Simon [15], Greenlees and May [8], Tarrío, López and Lipman [17]. In [4], Cuong and Nam defined the local homology modules $H_{i}^{I}(M)$ of $M$ with respect to $I$ by

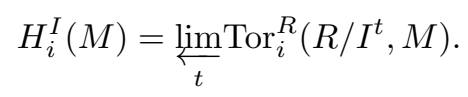

This concept in some sense is dual to Grothendiecks definition of local cohomology modules. Also, this definition of local homology modules coincides with the definition of Greenlees and May [8] when $M$ is an Artinian $R$-module.

The aim of this paper is to provide some properties of coregular sequences which are used in investigation top local homology modules. This work is inspired by some results in [2]. We see in Theorem 3.7 that if $M$ is a non-zero Artinian $R$-module and $\underline{x}:=x_{1}, \ldots, x_{n}$ is an $M$-coregular sequence, then $x_{1}, \ldots, x_{n}$ is a $D(H \underline{x}(M))$ coregular sequence. Let $M$ be an Artinian $R$-module of Noetherian dimension $n$ and $I$ an ideal of $R$, the top local homology module $H_{n}^{I}(M)$ is not finitely generated in general. Under condition $R$ is complete with respect to $I$-adic topology, we shall prove in Theorem 3.12 that $\operatorname{dim} H_{n}^{I}(M) \leq n$ and $\operatorname{depth} H_{I}^{n}(M) \geq \min \{2, n\}$ whenever $H_{n}^{I}(M) \neq 0$. 
Throughout, for any ideal $I$ of $R$, the set $\{m \in M \mid I m=0\}$ is denoted by $0:_{M} I$. For each $R$-module $M$, we denote by $\operatorname{Coass}_{R} M$ the set of all coassociated prime ideals of $M$ (see [18]). Also, we say that $R$ is complete with respect to $I$ adic topology if $R \cong H_{0}^{I}(R)$. If $(R, \mathfrak{m})$ is a local ring and $M$ is an $R$-module, then the Matlis duality of $M$ is denoted by $D(M)$. The projective dimension and flat dimension of $R$-module $M$ are denoted by $\operatorname{pd}_{R} M$ and $\mathrm{fd}_{R} M$, respectively. Many basic properties of local homology modules can be found in [4].

\section{Preliminaries}

First, we recall the definition of an $M$-coregular sequence. The concept of coregular sequence was introduced by Matlis [10] as a natural dual of the concept regular sequence.

Definition 2.1. ([10]) Let $M$ be an $R$-module. An element $x \in R$ is called coregular for $M$ (or $M$-coregular) if $M=x M$.

A sequence $x_{1}, x_{2}, \ldots, x_{n} \in R$ is called $M$-coregular sequence if

(i) $x_{i}$ is $0::_{M}\left(x_{1}, x_{2}, \ldots x_{i-1}\right)$-coregular for all $i=1,2, \ldots, n$;

(ii) $0::_{M}\left(x_{1}, x_{2}, \ldots x_{n}\right) \neq 0$.

We denote by $\operatorname{Width}_{I}(M)$ the supremum of the lengths of all maximal $M$ coregular sequences in $I$. If $M$ is an Artinian $R$-module such that $0:_{M} I \neq 0$, then we see in [13] that

$$
\operatorname{Width}_{I}(M)=\inf \left\{i \mid \operatorname{Tor}_{i}^{R}(R / I, M) \neq 0\right\}<\infty .
$$

Moreover, it follows from [6] that

$$
\operatorname{Width}_{I}(M)=\inf \left\{i \mid H_{i}^{I}(M) \neq 0\right\} .
$$

We now recall the concept of Noetherian dimension $\operatorname{Nim} M$ of an $R$-module which was introduced by Roberts [14] with the terminology Krull dimension. Kirby

[9] changed the terminology of Roberts and referred to Noetherian dimension to avoid confusion with the well-known Krull dimension for finitely generated modules.

Definition 2.2. ([14]) The Noetherian dimension of $M$, denoted by $\mathrm{N} \operatorname{dim} M$, is defined inductively as follows.

When $M=0$ we define $\mathrm{N} \operatorname{dim} M=-1$. Then by induction, for any integer $r \geq 0$, we define $\operatorname{Ndim} M=r$ when

(i) $\operatorname{Nim} M<r$ is false, and

(ii) for every ascending chain $M_{1} \subseteq M_{2} \subseteq$. of submodules of $M$ there exists an integer $n_{0}$ such that $\operatorname{Ndim}\left(M_{n+1} / M_{n}\right)<r$ for all $n \geq n_{0}$. 
Thus $M$ is non-zero and finitely generated if and only if $\operatorname{Ndim} M=0$. If $M$ is Artinian module, then $\operatorname{Ndim} M<\infty$ (see [14]). Also, if $M$ is an Artinian $R$-module with $\operatorname{Ndim} M=d>0$ and $x \in R$ is an $M$-coregular element, then $\operatorname{Ndim}\left(0:_{M} x\right)=$ $d-1$ (see [7]). Cuong and Nam [4] showed that if $M$ is an Artinian $R$-module, then $H_{i}^{I}(M)=0$ for all $i>\operatorname{Nim} M$. Moreover, if $(R, \mathfrak{m})$ is a local ring and $M$ is non-zero, then $H_{\mathrm{Ndim} M}^{\mathfrak{m}}(M) \neq 0$.

In [16], the authors introduced the concept of Co-Cohen-Macaulay modules by comparing the Noetherian dimension and width for Artinian modules over a local ring.

Definition 2.3. Let $(R, \mathfrak{m})$ be a local ring. An Artinian module $M$ is called CoCohen-Macaulay if $\operatorname{Ndim} M=\operatorname{Width}_{\mathfrak{m}}(M)$.

It is clear that if $M$ is a non-zero Co-Cohen-Macaulay $R$-module, then $H_{i}^{\mathfrak{m}}(M)=$ 0 for all $i \neq \operatorname{Nim} M$.

\section{Results}

First, we will study the coregular sequences in connection with local homology modules and homological functor.

Proposition 3.1. Let $M$ be a non-zero Artinian $R$-module and $\underline{x}:=x_{1}, \ldots, x_{n}$ a maximal $M$-coregular sequence. Then

$$
\operatorname{Tor}_{i}^{R}\left(R / \underline{x}, H \frac{x}{n}(M)\right) \cong \operatorname{Tor}_{n+i}^{R}(R / \underline{x}, M)
$$

for all $i \geq 0$.

Proof. It follows from [12, Lemma 3.1(i)] that there is a Grothendieck spectral sequence

$$
E_{p, q}^{2}=\operatorname{Tor}_{p}^{R}\left(R / \underline{x}, H \frac{x}{q}(M)\right) \underset{p}{\Rightarrow} \operatorname{Tor}_{p+q}^{R}(R / \underline{x}, M) .
$$

Let $i \geq 0$ an integer, there exists a filtration $\Phi$ of submodules of $H_{n+i}=\operatorname{Tor}_{n+i}^{R}(R / \underline{x}, M)$

$$
0=\Phi^{-1} H_{n+i} \subseteq \ldots \subseteq \Phi^{n+i-1} H_{n} \subseteq \Phi^{n+i} H_{n+i}=H_{n+i}
$$

such that

$$
E_{j, n+i-j}^{\infty} \cong \Phi^{j} H_{n+i} / \Phi^{j-1} H_{n+i}
$$

for all $0 \leq j \leq n+i$. It should be aware that $H_{i}^{\underline{x}}(M)=0$ for all $i \neq n$. This indicates $E_{p, q}^{\infty}=0$ for all $p \geq 0, q \neq n$ and $E_{i, n}^{2}=E_{i, n}^{\infty}$ for all $i \geq 0$. Consequently, one gets that

$$
\Phi^{i-1} H_{n+i}=\ldots=\Phi^{0} H_{n+i}=\Phi^{-1} H_{n+i}=0
$$


and

$$
\Phi^{i} H_{n+i}=\ldots=\Phi^{i+n} H_{n+i}=H_{n+i} .
$$

Thus, there is an isomorphism $\operatorname{Tor}_{i}^{R}\left(R / \underline{x}, H_{n}^{\underline{x}}(M)\right) \cong \operatorname{Tor}_{n+i}^{R}(R / \underline{x}, M)$, as required.

In [18], a prime ideal $\mathfrak{p}$ is called coassociated to a non-zero $R$-module $M$ if there is an Artinian homomorphic image $T$ of $M$ with $\mathfrak{p}=\operatorname{Ann} T$. The set of coassociated primes of $M$ is denoted by $\operatorname{Coass}_{R} M$.

Corollary 3.2. Let $M$ be a non-zero Artinian $R$-module and $\underline{x}:=x_{1}, \ldots, x_{n} a$ maximal $M$-coregular sequence. Then $\operatorname{Coass}_{R} H_{n}^{x}(M)$ is a finite set.

Proof. Proposition 3.1 shows that

$$
\operatorname{Tor}_{0}^{R}(R / \underline{x}, H \underline{x}(M)) \cong \operatorname{Tor}_{n}^{R}(R / \underline{x}, M) .
$$

Combining [5, Theorem 4.2(i)] with [5, Corollary 3.4], we realize that $\operatorname{Coass}_{R} H_{n}^{x}(M) \subseteq$ $V(\underline{x})$. By [18, Theorem 1.21],

$$
\begin{aligned}
\operatorname{Coass}_{R}\left(\operatorname{Tor}_{0}^{R}\left(R / \underline{x}, H \frac{x}{n}(M)\right)\right) & =V(\underline{x}) \cap \operatorname{Coass}_{R} H \frac{x}{n}(M) \\
& =\operatorname{Coass}_{R} H \underline{x}(M) .
\end{aligned}
$$

Since $M$ is an Artinian $R$-module, the set $\operatorname{Coass}_{R} \operatorname{Tor}_{n}^{R}(R / \underline{x}, M)$ is finite. Thus, the assertion follows from the above isomorphism.

If $M$ is a finitely generated module over a local ring $(R, \mathfrak{m})$, then we know that

$$
\operatorname{pd}_{R} M=\sup \left\{i \mid \operatorname{Tor}_{i}^{R}(R / \mathfrak{m}, M) \neq 0\right\} .
$$

In the case where $M$ is an Artinian $R$-module, the flat dimension of $M$ is defined as (see [1])

$$
\mathrm{fd}_{R} M=\sup \left\{i \mid \operatorname{Tor}_{i}^{R}(R / \mathfrak{m}, M) \neq 0\right\} .
$$

By using two above results, we have the following consequence.

Proposition 3.3. Let $(R, \mathfrak{m})$ be a complete local ring with respect to $\mathfrak{m}$-adic topology, $M$ a non-zero Artinian R-module. Assume that $x_{1}, \ldots, x_{n}$ is a maximal $M$ coregular sequence such that $\mathfrak{m}=\left(x_{1}, \ldots, x_{n}\right)$. Then the following statements hold:

(i) $\operatorname{pd}_{R} H_{n}^{\mathfrak{m}}(M)$ is finite if and only if $\mathrm{fd}_{R} M$ is finite.

(ii) If $\mathrm{fd}_{R} M<\infty$, then $\operatorname{pd}_{R} H_{n}^{\mathfrak{m}}(M)+n=\mathrm{fd}_{R} M$. 
Proof. It follows from [4, Proposition 4.6] that $H_{n}^{\mathfrak{m}}(M)$ is a finitely generated $R$ module. Combining the hypothesis with the isomorphism in Proposition 3.1, one obtains that

$$
\operatorname{Tor}_{i}^{R}\left(R / \mathfrak{m}, H_{n}^{\mathfrak{m}}(M)\right) \cong \operatorname{Tor}_{n+i}^{R}(R / \mathfrak{m}, M) .
$$

Consequently, the assertions follow from the above facts.

Before, stating and proving the first main result of this paper, we need some lemmas which provide us many properties of coregular sequences.

Lemma 3.4. Let $\underline{x}:=x_{1}, \ldots, x_{n} \in R$ be an $A$-coregular sequence and $0 \rightarrow A \stackrel{f}{\rightarrow}$ $B \stackrel{g}{\rightarrow} C \stackrel{h}{\rightarrow} D$ an exact sequence. Then there is an exact sequence

$$
0 \rightarrow 0:_{A} \underline{x} \rightarrow 0:_{B} \underline{x} \rightarrow 0:_{C} \underline{x} \rightarrow 0:_{D} \underline{x} .
$$

Proof. We need only consider the case where $x$ is an $A$-coregular element. Consider the chain of homomorphisms

$$
0 \rightarrow 0:_{A} x \stackrel{\bar{f}}{\rightarrow} 0:_{B} x \stackrel{\bar{g}}{\rightarrow} 0:_{C} x \stackrel{\bar{h}}{\rightarrow} 0:_{D} \underline{x},
$$

where $\bar{f}, \bar{g}, \bar{h}$ are restrictions of $f, g, h$ respectively. It remains to prove that the chain of homomorphisms is exact at $0:_{C} x$. Let $c \in \operatorname{Ker} \bar{h}$, then $0=\bar{h}(c)=h(c)$. There exists an element $b \in B$ such that $g(b)=c$ and $x g(b)=x c=0$. Therefore, we have $a \in A$ satisfying $f(a)=x b$. Since $x$ is an $A$-coregular element, one gets $a^{\prime} \in A$ such that $a=x a^{\prime}$. This implies that $b-f\left(a^{\prime}\right) \in 0:_{B} x$ and $\bar{g}\left(b-f\left(a^{\prime}\right)\right)=c$. Consequently, we have $c \in \operatorname{Im} \bar{g}$, and the proof is complete.

It should be pointed out that $0::_{A} \underline{x} \cong \operatorname{Hom}(R / \underline{x}, M)$. Therefore, we immediately have a consequence.

Corollary 3.5. Let $\underline{x}:=x_{1}, \ldots, x_{n} \in R$ be an $M$-coregular sequence. Then

$$
\operatorname{Ext}_{R}^{1}(R / \underline{x}, M)=0 \text {. }
$$

Combining [11, Proposition 1.1.7] with [5, Proposition 4.1], there is a MayerVietoris sequence.

Lemma 3.6. Let $I, J$ be two ideals of $R$ and $M$ an Artinian $R$-module. Then there is an exact sequence

$$
\ldots \rightarrow H_{i}^{I \cap J}(M) \rightarrow H_{i}^{I}(M) \oplus H_{i}^{J}(M) \rightarrow H_{i}^{I+J}(M) \rightarrow H_{i-1}^{I \cap J}(M) \rightarrow \cdots .
$$

Now, the first main result relates to the Matlis duality of top local homology modules and coregular sequences. 
Theorem 3.7. Let $M$ be a non-zero Artinian $R$-module. Assume that $\underline{x}:=$ $x_{1}, \ldots, x_{n}$ is an $M$-coregular sequence. Then $x_{1}, \ldots, x_{n}$ is a $D(H \bar{x}(M))$-coregular sequence.

Proof. The proof is by induction on $n$. Let $n=1$, the short exact sequence

$$
0 \rightarrow 0: M x_{1} \rightarrow M \stackrel{x_{1}}{\rightarrow} M \rightarrow 0
$$

induces the following exact sequence

$$
0 \rightarrow H_{1}^{\left(x_{1}\right)}\left(0:_{M} x_{1}\right) \rightarrow H_{1}^{\left(x_{1}\right)}(M) \stackrel{x_{1}}{\rightarrow} H_{1}^{\left(x_{1}\right)}(M) \rightarrow H_{0}^{\left(x_{1}\right)}\left(0:_{M} x_{1}\right) \rightarrow 0 .
$$

It is clear that $H_{0}^{\left(x_{1}\right)}\left(0:_{M} x_{1}\right) \cong 0:_{M} x_{1}$ and $H_{1}^{\left(x_{1}\right)}\left(0:_{M} x_{1}\right)=0$. Hence, there is a short exact sequence

$$
0 \rightarrow H_{1}^{\left(x_{1}\right)}(M) \stackrel{x_{1}}{\rightarrow} H_{1}^{\left(x_{1}\right)}(M) \rightarrow 0:_{M} x_{1} \rightarrow 0
$$

which yields the following exact sequence

$$
0 \rightarrow D\left(0:_{M} x_{1}\right) \rightarrow D\left(H_{1}^{\left(x_{1}\right)}(M)\right) \stackrel{x_{1}}{\rightarrow} D\left(H_{1}^{\left(x_{1}\right)}(M)\right) \rightarrow 0 .
$$

Since $x_{1}$ is an $M$-coregular sequence, if follows that $D\left(0:_{M} x_{1}\right) \neq 0$. Thus, $x_{1}$ is a $D\left(H_{1}^{\left(x_{1}\right)}(M)\right)$-coregular sequence.

Now, assume that $n>1$ and the claim is true for the cases are less than $n$. The short exact sequence

$$
0 \rightarrow 0: M x_{1} \rightarrow M \stackrel{x_{1}}{\rightarrow} M \rightarrow 0
$$

leads the long exact sequence

$$
0 \rightarrow H_{\bar{n}}^{\underline{x}}\left(0:_{M} x_{1}\right) \rightarrow H_{\bar{n}}^{x}(M) \stackrel{x_{7}}{\rightarrow} H_{\bar{n}}^{\underline{x}}(M) \rightarrow H_{n-1}^{\underline{x}}\left(0:_{M} x_{1}\right) \rightarrow 0 .
$$

Lemma 3.6 gives us a long exact sequence

$$
\ldots H_{n}^{I}\left(0:_{M} x_{1}\right) \oplus H_{n}^{J}\left(0:_{M} x_{1}\right) \rightarrow H_{n}^{I+J}\left(0:_{M} x_{1}\right) \rightarrow H_{n-1}^{I \cap J}\left(0:_{M} x_{1}\right) \rightarrow \cdots,
$$

where $I=\left(x_{1}\right)$ and $J=\left(x_{2}, \ldots, x_{n}\right)$. It is clear that $H_{i}^{I}\left(0:_{M} x_{1}\right)=0$ for all $i>1$. By [6, Theorem 3.8], we see that $H_{i}^{I \cap J}\left(0:_{M} x_{1}\right)=0$ for all $i>0$. Hence, there is an isomorphism

$$
H_{i}^{\left(x_{2}, \ldots, x_{n}\right)}\left(0:_{M} x_{1}\right) \cong H_{i}^{\left(x_{1}, \ldots, x_{n}\right)}\left(0:_{M} x_{1}\right)
$$

for all $i \geq 2$. This implies that

$$
H_{n}^{\left(x_{1}, \ldots, x_{n}\right)}\left(0:_{M} x_{1}\right) \cong H_{n}^{\left(x_{2}, \ldots, x_{n}\right)}\left(0:_{M} x_{1}\right)=0 .
$$

Consequenlty, there is a short exact sequence

$$
0 \rightarrow H \underline{x}(M) \stackrel{x_{1}}{\rightarrow} H_{n}^{\underline{x}}(M) \rightarrow H_{n-1}^{\underline{x}}\left(0:_{M} x_{1}\right) \rightarrow 0
$$


which induces another exact sequence

$$
0 \rightarrow D\left(H_{n-1}^{\underline{x}}\left(0:_{M} x_{1}\right)\right) \rightarrow D\left(H \frac{x}{n}(M)\right) \stackrel{x_{1}}{\rightarrow} D\left(H \frac{x}{n}(M)\right) \rightarrow 0 .
$$

The inductive hypothesis shows that $x_{2}, \ldots, x_{n}$ is a $D\left(H_{n-1}^{\underline{x}}\left(0:_{M} x_{1}\right)\right)$-coregular sequence. Hence, $x_{2}, \ldots, x_{n}$ is a $\left(0:_{D\left(H \frac{x}{n}(M)\right)} x_{1}\right)$-coregular sequence and the assertion follows.

The following result is a property of coregular sequences.

Corollary 3.8. Let $M$ be a non-zero Artinian R-module. Let $\underline{x}:=x_{1}, \ldots, x_{n}$ be an $M$-coregular sequence. Then

$$
\operatorname{Tor}_{n+1}^{R}(R / \underline{x}, M)=0 .
$$

Proof. By Proposition 3.1, there is an isomorphism

$$
\operatorname{Tor}_{n+1}^{R}(R / \underline{x}, M) \cong \operatorname{Tor}_{1}^{R}\left(R / \underline{x}, H_{\bar{n}}^{x}(M)\right)
$$

which induces

$$
D\left(\operatorname{Tor}_{n+1}^{R}(R / \underline{x}, M)\right) \cong \operatorname{Ext}_{R}^{1}\left(R / \underline{x}, D\left(H_{\bar{n}}^{x}(M)\right)\right) .
$$

Combining Corollary 3.5 with Theorem 3.7, we get

$$
D\left(\operatorname{Tor}_{n+1}^{R}(R / \underline{x}, M)\right)=0,
$$

and which completes the proof.

Theorem 3.9. Let $(R, \mathfrak{m})$ be a local ring and $M$ a non-zero Co-Cohen-Macaulay $R$ module with $\operatorname{Ndim} M=d>0$. Assume that $\underline{x}:=x_{1}, \ldots, x_{n} \in \mathfrak{m}$ is an $M$-coregular sequence. Then $x_{1}, \ldots, x_{n}$ is a $H_{d}^{\mathfrak{m}}(M)$-regular sequence.

Proof. The proof is by induction on $n$. Let $n=1$, the short exact sequence

$$
0 \rightarrow 0: M x_{1} \rightarrow M \stackrel{x_{7}}{\rightarrow} M \rightarrow 0
$$

yields the long exact sequence

$$
0 \rightarrow H_{d}^{\mathfrak{m}}\left(0:_{M} x_{1}\right) \rightarrow H_{d}^{\mathfrak{m}}(M) \stackrel{x_{7}}{\rightarrow} H_{d}^{\mathfrak{m}}(M) \rightarrow H_{d-1}^{\mathfrak{m}}\left(0:_{M} x_{1}\right) \rightarrow 0 .
$$

By [16, Lemma 2.2 (ii)], we see that $\operatorname{Ndim}\left(0:_{M} x_{1}\right)=d-1$ and then $H_{d}^{\mathfrak{m}}\left(0:_{M}\right.$ $\left.x_{1}\right)=0$ by $\left[4\right.$, Proposition 4.8]. It follows from [4, Proposition 4.10] that $H_{d-1}^{\mathfrak{m}}\left(0:_{M}\right.$ $\left.x_{1}\right) \neq 0$. This means that $x_{1}$ is a $H_{d}^{\mathfrak{m}}(M)$-regular sequence.

Now, assume that $n>1$. The short exact sequence

$$
0 \rightarrow 0::_{M} x_{1} \rightarrow M \stackrel{x_{7}}{\rightarrow} M \rightarrow 0
$$


yields the long exact sequence

$$
0 \rightarrow H_{d}^{\mathfrak{m}}(M) \stackrel{x_{1}}{\rightarrow} H_{d}^{\mathfrak{m}}(M) \rightarrow H_{d-1}^{\mathfrak{m}}\left(0:_{M} x_{1}\right) \rightarrow 0 \text {. }
$$

It should be mentioned that $0:_{M} x_{1}$ is a Co-Cohen-Macaulay $R$-module with $\operatorname{Ndim}\left(0::_{M} x_{1}\right)=d-1$. Note that $x_{2}, \ldots, x_{n}$ is $0:_{M} x_{1}$-coregular sequence. By the inductive hypothesis, it is a $H_{d-1}^{\mathfrak{m}}\left(0::_{M} x_{1}\right)$-regular sequence and the claim follows.

Proposition 3.10. Let $(R, \mathfrak{m})$ be a local ring, $M$ a non-zero Co-Cohen-Macaulay $R$-module with $\operatorname{Ndim} M=d$. Assume that $R$ is complete with respect to $\mathfrak{m}$-adic topology. Then depth $H_{d}^{\mathfrak{m}}(M)=d$.

Proof. We prove by induction on $d$. If $d=0$, then $M$ has finite length. It is clear that $H_{0}^{\mathfrak{m}}(M) \cong M$ and $\operatorname{depth} H_{0}^{\mathfrak{m}}(M)=0$.

Assume that $d>0$ and $x_{1}, \ldots, x_{d}$ is a maximal $M$-coregular sequence in $\mathfrak{m}$. By the similar method in the proof of Theorem 3.7, one has

$$
\operatorname{depth} H_{d}^{\mathfrak{m}}(M) / x_{1} H_{d}^{\mathfrak{m}}(M)=\operatorname{depth} H_{d-1}^{\mathfrak{m}}\left(0:_{M} x_{1}\right)=d-1
$$

Combining the assumption with [4, Proposition 4.6], we see that $H_{d}^{\mathfrak{m}}(M)$ is finitely generated $R$-module. Hence, [3, Proposition 1.2.10 (d)] shows that

$$
\operatorname{depth} H_{d}^{\mathfrak{m}}(M) / x_{1} H_{d}^{\mathfrak{m}}(M)=\operatorname{depth} H_{d}^{\mathfrak{m}}(M)-1 \text {, }
$$

and which completes the proof.

Corollary 3.11. Let $(R, \mathfrak{m})$ be a local ring, $M$ a non-zero Co-Cohen-Macaulay $R$-module with $\operatorname{Ndim} M=d$. Assume that $R$ is complete with respect to $\mathfrak{m}$-adic topology and $x_{1}, \ldots, x_{d} \in \mathfrak{m}$ is an $M$-coregular sequence such that $\mathfrak{m}=\left(x_{1}, \ldots, x_{d}\right)$. Then $\operatorname{fd}_{R} M=\operatorname{depth} R$.

Proof. Using Auslander-Buchsbaum formula, Proposition 3.3 (ii) and Proposition 3.10 , we get the claim.

Theorem 3.12. Let $(R, \mathfrak{m})$ be a local ring and $M$ a non-zero Artinian $R$-module with $\mathrm{N} \operatorname{dim} M=d$. Assume that $R$ is complete with respect to I-adic topology. Then the following statements hold:

(i) $\operatorname{dim} H_{d}^{I}(M) \leq d$;

(ii) If $H_{d}^{I}(M) \neq 0$, then $\operatorname{depth} H_{d}^{I}(M) \geq \min \{2, d\}$.

Proof. We use induction on $d$. Let $d=0$, then $M$ has finite length and $H_{0}^{I}(M) \cong$ $M$. Consequently, one gets $\operatorname{dim} H_{0}^{I}(M)=0$ and $\operatorname{depth} H_{0}^{I}(M)=0$. 
Let $d>0$. By [4, Proposition 4.4 and Corollary 4.5], we may assume that there exists $x \in \mathfrak{m}$ which is an $M$-coregular element. It was shown in [16, Lemma 2.2] that $\operatorname{Nim}\left(0:_{M} x\right)=d-1$. The short exact sequence

$$
0 \rightarrow 0: M x \stackrel{x}{\rightarrow} M \rightarrow 0
$$

induces a long exact sequence

$$
0 \rightarrow H_{d}^{I}(M) \stackrel{x}{\rightarrow} H_{d}^{I}(M) \rightarrow H_{d-1}^{I}\left(0:_{M} x\right) \rightarrow \cdots
$$

Since $H_{d}^{I}(M) / x H_{d}^{I}(M)$ is isomorphic to a submodule of $H_{d-1}^{I}\left(0:_{M} x\right)$, it follows from the hypothesis that

$$
\operatorname{dim} H_{d}^{I}(M) / x H_{d}^{I}(M) \leq d-1
$$

In view of [6, Theorem 5.3] and the hypothesis, we can conclude that $H_{d}^{I}(M)$ is a finitely generated $R$-module. This implies that

$$
\operatorname{dim} H_{d}^{I}(M) / x H_{d}^{I}(M)=\operatorname{dim} H_{d}^{I}(M)-1
$$

and then $\operatorname{dim} H_{d}^{I}(M) \leq d$.

It induces from the Nakayama's Lemma and the assumption that $H_{d}^{I}(M) / x H_{d}^{I}(M) \neq 0$. This indicates that $H_{d-1}^{I}\left(0::_{M} x\right) \neq 0$. Consequently, if $d=1$, then we see that $\operatorname{depth} H_{1}^{I}(M)=1$. We consider the cases where $d \geq 2$. By the inductive hypothesis, one can claim that $\operatorname{depth} H_{d-1}^{I}\left(0:_{M} x\right) \geq 1$. Let $y$ be a $H_{d-1}^{I}\left(0:_{M} x\right)$-regular element. It is easy to check that $y$ is a $H_{d}^{I}(M) / x H_{d}^{I}(M)$ regular element. Thus, we can assert that $\operatorname{depth} H_{d}^{I}(M) \geq \min \{2, d\}$.

Corollary 3.13. Let $(R, \mathfrak{m})$ be a local ring, $M$ a non-zero Co-Cohen-Macaulay $R$-module with $\operatorname{Ndim} M=d$. Assume that $R$ is complete with respect to $\mathfrak{m}$-adic topology. Then $H_{d}^{\mathfrak{m}}(M)$ is a Cohen-Macaulay R-module of dimension $d$.

Proof. Combining Proposition 3.10 with Theorem 3.12, the assertion follows.

Corollary 3.14. Let $(R, \mathfrak{m})$ be a local ring, $M$ a non-zero Artinian $R$-module with $\operatorname{Ndim} M=d \leq 2$. Assume that $R$ is complete with respect to I-adic topology and $H_{d}^{I}(M) \neq 0$. Then $H_{d}^{I}(M)$ is Cohen-Macaulay.

Acknowledgement. The author would like to thank the referee for the valuable suggestions and comments. 


\section{References}

[1] K. Bahmanpour, A note on homological dimensions of Artinian local cohomology modules, Canad. Math. Bull., 56(3) (2013), 491-499.

[2] K. Bahmanpour, A complex of modules and its applications to local cohomology and extension functors, Math. Scand., 117(1) (2015), 150-160.

[3] W. Bruns and J. Herzog, Cohen-Macaulay Rings, Cambridge University Press, 1993.

[4] N. T. Cuong and T. T. Nam, The I-adic completion and local homology for Artinian modules, Math. Proc. Cambridge Philos. Soc., 131(1) (2001), 61-72.

[5] N. T. Cuong and T. T. Nam, On the co-localization, co-support and coassociated primes of local homology modules, Vietnam J. Math., 29(4) (2001), 359-368.

[6] N. T. Cuong and T. T. Nam, A local homology theory for linearly compact modules, J. Algebra, 319(11) (2008), 4712-4737.

[7] N. T. Cuong and L. T. Nhan, On the Noetherian dimension of Artinian modules, Vietnam J. Math., 30(2) (2002), 121-130.

[8] J. P. C. Greenlees and J. P. May, Derived functors of I-adic completion and local homology, J. Algebra, 149(2) (1992), 438-453.

[9] D. Kirby, Dimension and length for Artinian modules, Quart. J. Math. Oxford Ser.(2), 41(164) (1990), 419-429.

[10] E. Matlis, The Koszul complex and duality, Comm. Algebra, 1 (1974), 87-144.

[11] T. T. Nam, On local homology theory for linearly compact modules, Ph.D. Thesis, Institute of Mathematics, Hanoi, 2001.

[12] T. T. Nam, On the finiteness of co-associated primes of local homology modules, J. Math. Kyoto Univ., 48(3) (2008), 521-527.

[13] A. Ooishi, Matlis duality and the width of a module, Hiroshima Math. J., 6(3) (1976), 573-587.

[14] R. N. Roberts, Krull dimension for Artinian modules over quasi local commutative rings, Quart. J. Math. Oxford Ser. (2), 26(103) (1975), 269-273.

[15] A-M. Simon, Some homological properties of complete modules, Math. Proc. Cambridge Philos. Soc., 108(2) (1990), 231-246.

[16] Z. Tang and H. Zakeri, Co-cohen-macaulay modules and modules of generalized fractions, Comm. Algebra, 22(6) (1994), 2173-2204.

[17] A. A. Tarrío, A. J. López and J. Lipman, Local homology and cohomology on schemes, Ann. Sci. cole Norm. Sup. (4), 30(1) (1997), 1-39.

[18] S. Yassemi, Coassociated primes, Comm. Algebra, 23(4) (1995), 1473-1498. 
Nguyen Minh Tri

Department of Mathematics and Physics

University of Information Technology

Vietnam National University Ho Chi Minh City

Quarter 6, Linh Trung Ward, Thu Duc City, Ho Chi Minh City, Vietnam

e-mail: trinm@uit.edu.vn, triminhng@gmail.com 